\title{
Evidence for a three-nucleon-force effect in proton-deuteron elastic scattering
}

\author{
R. V. Cadman ${ }^{1}$, J. Brack ${ }^{2}$, W. J. Cummings ${ }^{3}$, J. A. Fedchak ${ }^{3}$, B. D. Fox ${ }^{2}$, \\ H. Gao ${ }^{4}$, W. Glöckle ${ }^{5}$, C. Grosshauser ${ }^{6}$, R. J. Holt ${ }^{1}$, C. E. Jones ${ }^{7}$, E. R. Kinney ${ }^{2}$, \\ M. A. Miller ${ }^{1}$, W. Nagengast ${ }^{6}$, B. R. Owen ${ }^{1}$, K. Rith ${ }^{6}$, F. Schmidt ${ }^{6}$, E. C. Schulte ${ }^{1}$,

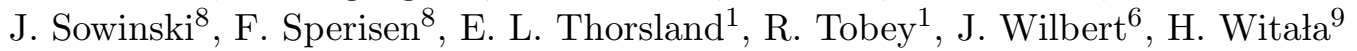 \\ ${ }^{1}$ Department of Physics, University of Illinois, Urbana, Illinois 61801 \\ ${ }^{2}$ Nuclear Physics Laboratory, University of Colorado, Boulder, Colorado \\ 80309-0446 \\ ${ }^{3}$ Physics Division, Argonne National Laboratory, Argonne, Illinois 60439 \\ ${ }^{4}$ Laboratory for Nuclear Science, Massachusetts Institute of Technology, \\ Cambridge, Massachusetts 02139 \\ ${ }^{5}$ Institut für theoretische Physik II, Ruhr-Universität Bochum, D-44780 Bochum, \\ Germany \\ ${ }^{6}$ Physikalisches Institut, Universität Erlangen-Nürnberg, D-91058 Erlangen, \\ Germany \\ ${ }^{7}$ W.K. Kellogg Radiation Lab, California Institute of Technology, Pasadena, \\ California 91125 \\ ${ }^{8}$ Indiana University Cyclotron Facility, Bloomington, Indiana 47408 \\ ${ }^{9}$ Institute of Physics, Jagellonian University, PL-30059 Cracow, Poland
}

\begin{abstract}
Developments in spin-polarized internal targets for storage rings have permitted measurements of $197 \mathrm{MeV}$ polarized protons scattering from vector polarized deuterons. This work presents measurements of the polarization observables $A_{y}, i T_{11}$, and $C_{y, y}$ in proton-deuteron elastic scattering. When compared to calculations with and without three-nucleon forces, the measurements indicate that three-nucleon forces make a significant contribution to the observables. This work indicates that three-body forces derived from static nuclear properties appear to be crucial to the description of dynamical properties.
\end{abstract}

Understanding how nuclei are built from their constituent protons and neutrons and the forces between them is one of the fundamental goals of nuclear physics. An important aspect of the nuclear force is the modification which occurs as nucleons become embedded in the nuclear medium. The three-nucleon system provides an important laboratory because the Schrödinger equation, in the form of Faddeev equations, can be solved exactly for three bodies. New computational capabilities have extended the range of validity for Faddeev calculations to a wide range of kinematics, from bound states to scattering and three-body breakup at energies up to $200 \mathrm{MeV}$.

Modern two-nucleon potentials based on the exchange of pions and heavier mesons [1] 3] provide an excellent description of neutron-proton and proton-proton 
scattering data and of the deuteron. In contrast, it is now generally accepted that the binding energies of other light nuclei cannot be calculated from the modern two-nucleon potentials alone [1, 1 , 6]. The only successful resolution of this problem has been to include additional potential terms which act only in the presence of at least three nucleons [7, 8]. These terms, known as three-nucleon forces (3NF), are also essential for understanding nuclear matter in extreme conditions, such as dense nuclear matter and neutron stars [9].

Three-body forces are expected in nuclear physics because conventional nuclear theory is a simplification of the fundamental theory of the strong interaction, quantum chromodynamics. In conventional nuclear theory, the nucleons are treated as fundamental particles, and the nucleon excited states, which are an expression of the underlying quark degrees of freedom, are not explicitly included. The addition of nucleon excited states leads to forces which cannot be reduced to successive twonucleon interactions. For example, a pion exchange between two nucleons can excite one nucleon two a $\Delta$ which subsequently decays by pion exchange with a third nucleon. That process was included in the first three-nucleon potential, proposed by Fujita and Miyazawa in 1957 [10]. Modern three-nucleon potentials are more extensive, and include terms which follow from a fundamental symmetry of the strong interaction, chiral symmetry 11.

Although the binding energies imply that the $3 \mathrm{NF}$ is significant, they only constrain its overall strength. An investigation of nucleon-deuteron $(N d)$ scattering is needed to study the dynamical characteristics of the three-body force. For example, since the momentum transferred to the deuteron can be varied by changing the incident nucleon energy and the scattering angle, it is possible to probe the spatial dependence of the $3 \mathrm{NF}$ by using this reaction. Recent calculations have shown that the Tucson-Melbourne (TM) 3NF [7] predicts a significantly enhanced differential cross section in $N d$ elastic scattering when the kinetic energy of the incident nucleon is greater than about $60 \mathrm{MeV}$ 12.

Previous work compared measurements of the proton analyzing power $A_{y}$ in $\vec{p} \vec{d}$ elastic scattering to Faddeev calculations [13]. These authors showed that for a deuteron recoil angle $\theta_{\text {lab }}=42.6^{\circ}$ and over a proton energy range from 120 to $200 \mathrm{MeV}$, the Tucson-Melbourne potential over-corrects the prediction of the CDBonn potential. They also showed earlier measurements of $A_{y}$ at $200 \mathrm{MeV}$ 14, 15, which demonstrate that neither theory predicts the correct angular dependence for $A_{y}$. Another recent measurement showed the same result at $150 \mathrm{MeV}$ [20]. This indicates that the $A_{y}$ puzzle [16] first observed at low energies [17] persists at higher energies. The discrepancy in $A_{y}$ at low energies is believed to be due in part to an uncertainty in the ${ }^{3} P_{J} n p$ phase shifts [18] or to the need for a tensor component in the three-nucleon force 19. Measurements of the deuteron analyzing power $i T_{11}$ at the equivalent of a proton energy of $135 \mathrm{MeV}$ [20] are in good agreement with the predictions of CD-Bonn+TM3NF. However, at that energy none of the predictions match the angular dependence of the tensor analyzing powers $A_{x x}$ and 
$A_{y y}$. At $200 \mathrm{MeV}$, the previous measurement of $i T_{11}$ [21] is not sensitive enough to distinguish the effect of the $3 \mathrm{NF}$. Preliminary measurements of the spin correlation parameter $C_{y, y}$ at $200 \mathrm{MeV}$ 22] were made at small angles $\left(\theta_{\mathrm{cm}}<60^{\circ}\right)$ where the Tucson-Melbourne potential does not contribute significantly to the observables.

In an effort to provide further constraints on the nuclear three-body force, we provide measurements of the three spin observables $A_{y}, i T_{11}$, and $C_{y, y}$ at a proton energy of $197 \mathrm{MeV}$ and throughout the angular range $65^{\circ}<\theta_{\mathrm{cm}}<115^{\circ}$, where the $3 \mathrm{NF}$ contribution is expected to be large. $C_{y, y}$ is a measure of the asymmetry between beam and target spins parallel and anti-parallel, $i T_{11}$ is a measure of the asymmetry associated with changing the sign of the deuteron's vector polarization, and $A_{y}$ is a measure of the asymmetry associated with changing the sign of the proton polarization. The present work made use of the polarized proton beam at the Indiana University Cyclotron Facility (IUCF) with a vector polarized deuteron target. Both the beam and target polarizations were parallel to the $y$ axis or perpendicular to the scattering plane. Both polarizations were changed regularly and independently, so that the three different polarization observables could be measured simultaneously.

The proton beam used in this experiment was accelerated to $197 \mathrm{MeV}$ by IUCF's two cyclotrons and then stored in an electron-cooled proton storage ring. The average beam current was about $75 \mu \mathrm{A}$. The beam spin direction was alternated between parallel and anti-parallel to $\hat{\mathbf{y}}$ each time the ring was filled.

The experimental apparatus is shown schematically in Figure 1. The detectors were symmetric about the beam line, and included a forward thin scintillator $(0.31$ cm thick), two delay-line wire chambers, and a stack of three $100 \mathrm{~cm} \times 15 \mathrm{~cm} \times 10$ $\mathrm{cm}$ scintillator bars. The wire chambers consisted of two planes of wires, with wires running horizontal in one plane and vertical in the other. The wire spacing was $0.8 \mathrm{~cm}$, which led to an angular resolution of about $15 \mathrm{mr}$. The momentum of the scattered protons and deuterons was measured from the time of flight between the thin scintillator and the thick scintillator array. Deuterons were distinguished from protons by the relationship between the time of flight and the energy deposited in the thick scintillators.

Polarized deuterium atoms were injected into the center of the target, a $40 \mathrm{~cm} \times$ $3.2 \mathrm{~cm} \times 1.3 \mathrm{~cm}$ rectangular aluminum tube open at both ends to allow the proton beam to pass. The polarization of the target was continuously monitored using a Rabi polarimeter which sampled atoms from the center of the target cell. The magnetic field in the target cell was directed along the $y$ axis, and its magnitude ranged from $70 \mathrm{mT}$ in the center to $40 \mathrm{mT}$ at the ends.

A more complete description of the laser-driven polarized deuterium source used in this experiment can be found elsewhere [23]. The main points are summarized here. Deuterium molecules were dissociated and the resulting atoms were mixed with a potassium vapor in a spin-exchange cell. The valence electrons of the potassium atoms were polarized by optical pumping with circularly-polarized light from 


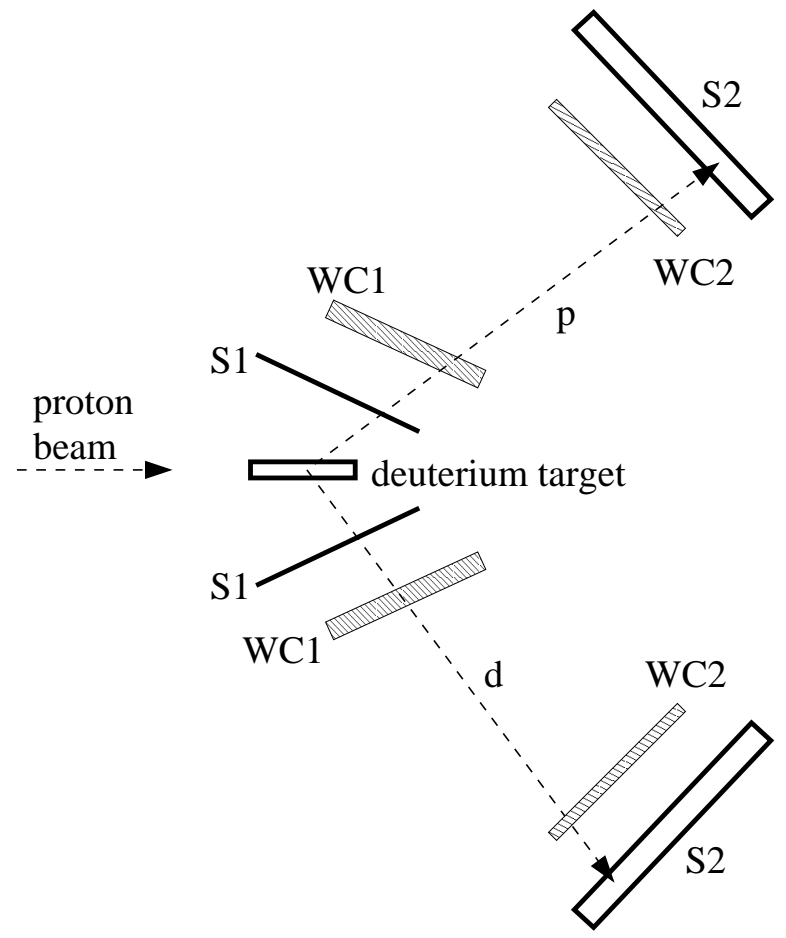

Figure 1: The experimental apparatus, viewed from above. The detectors are arranged symmetrically about the beam line. Each side has a forward thin scintillator (S1), two delay-line wire chambers (WC1 and WC2), and a stack of three thick scintillators (S2). Possible trajectories of an outgoing proton (p) and deuteron (d) are also shown. Not shown are the polarized source above the target cell and the atomic polarimeter below.

a titanium-sapphire laser. The target polarization was reversed at intervals of about 30 seconds by switching the helicity of the laser light and slightly retuning the laser to the appropriate frequency. A $70 \mathrm{mT}$ magnetic field in the cell inhibited radiation trapping in the potassium vapor. The magnetic field was parallel to the $y$ axis and defined the polarization axis for the source. The deuterium electrons were polarized by collisions between deuterium and potassium atoms in which the spins of the valence electrons in the two atoms are exchanged. This spin-exchange mechanism also polarized the deuterium nuclei through deuterium-deuterium (D-D) collisions as a result of the small mixing between the electron and nuclear spins still present at $70 \mathrm{mT}$ [24]. The D-D spin-exchange distributed angular momentum between the electrons and nuclei, bringing the system toward a state of spin-temperature equilibrium. Previous work verified that spin-temperature equilibrium is reached in laser-driven hydrogen [25] and deuterium [26] sources.

The most accurate measurement of the beam polarization was obtained by using 
a previous measurement of $A_{y}$ in $\vec{p} d$ elastic scattering at $198.6 \mathrm{MeV}$ proton energy and at a recoil deuteron lab angle of $42.6^{\circ}$ [13]. The beam polarization has a relative statistical error of $1.1 \%$, and the previous measurement had a total error of $0.4 \%$. The different beam energies in the two experiments contribute an error of $0.3 \%$. Added in quadrature, these errors give an overall relative error of $1.2 \%$ in the normalization of $A_{y}$. During the experiment, the beam polarization varied between 0.61 and 0.75 .

The target polarization was determined by measuring the polarization asymmetries in the deuteron breakup reaction. In the plane-wave impulse approximation, the spin observables for proton knockout are equal to the well-known spin observables for $\vec{p} \vec{p}$ elastic scattering at the center of mass energy of the two protons. A Monte Carlo calculation using a deuteron momentum wave function derived from the Argonne V18 potential [27] was used to determine the correction to the spin observables due to the momentum of the proton within the deuteron. The momentum of the outgoing neutron was restricted to $\left|\mathbf{p}_{n}\right|<60 \mathrm{MeV} /$ c so that the deuteron $D$ state contribution could be ignored. The results were not sensitive to the maximum neutron momentum. However, the beam polarization measured using this technique was approximately $13 \%$ below the value measured in the elastic reaction as described above. For this reason the relative systematic error in the target polarization was assigned to be $13 \%$.

The uncertainty in the target polarization is the dominant systematic error in $i T_{11}$ and $C_{y, y}$. Systematic errors in the angle reconstruction are less than $10 \mathrm{mr}$, and this leads to the largest systematic error in $A_{y}$ of \pm 0.007 . Errors due to particle identification, background subtraction, luminosity normalization, and beam energy have also been considered. The total systematic error from all sources other than the polarizations is \pm 0.010 for $A_{y}, \pm 0.011$ for $i T_{11}$, and \pm 0.015 for $C_{y, y}$.

The spin observables measured in this experiment have been compared to predictions both with and without the addition of a $3 \mathrm{NF}$. These predictions require a calculation of the transition amplitude for elastic nucleon-deuteron scattering, which includes the nucleon exchange term, the direct action of a $3 \mathrm{NF}$, and rescattering interactions of three nucleons through two- and three-nucleon forces. Details of the computational methods and performance have been published [28]. The CD-Bonn [1] and Argonne V18 [2] potentials were both used. Of the modern two-nucleon potentials, these two are believed to be the most different [29]. The $3 \mathrm{NF}$ was chosen to be the two-pion-exchange Tucson-Melbourne model. In the $3 \mathrm{NF}$, the strong cutoff parameter $\Lambda$ is adjusted separately for each two-nucleon potential to match the experimental triton binding energy [5]. The numerical error in solving the Schrödinger equation for $N d$ scattering is less than $2 \%$. The calculations were performed at an incident nucleon energy of $190 \mathrm{MeV}$. The resulting predictions are shown along with the experimental results in Figure 2 .

The top panel of Figure 2 shows the proton analyzing power $A_{y}$. These results are consistent with the previous measurements, and fall in between the two predictions. 
The center panel of Figure 2 shows the deuteron analyzing power $i T_{11}$. The pure twonucleon force calculations disagree with these results, but the calculations including the $3 \mathrm{NF}$ are consistent with the results. The bottom panel of Figure 2 shows the spin correlation parameter $C_{y, y}$. Again, the $2 \mathrm{NF}$ calculations disagree with the results at the largest angles, but the Argonne V18 calculation without the 3NF is consistent with the data at the smallest angles.

In conclusion, polarization observables in $\vec{p} \vec{d}$ elastic scattering have been measured at a proton energy of $197 \mathrm{MeV}$ over the angular range $65^{\circ}<\theta_{\mathrm{cm}}<115^{\circ}$. This was the first experiment to use a laser-polarized deuterium target. Calculations with the two-nucleon force alone do not reproduce the data, but the inclusion of the Tucson-Melbourne 3NF improves the agreement with data. These results provide evidence for the nuclear three-body force; however, further theoretical and experimental work is needed to fully characterize its spin dependence.

We thank J. L. Friar and V. R. Pandharipande for useful comments. We thank the staff and operators at IUCF, especially G. East, T. Sloan, R. Pollock, and J. Doskow, for their help and for the high quality of the beam we received. We also thank R. S. Kowalczyk, Z.-T. Lu, K. Bailey, G. Smith, and A. Kenyon for assisting with the development of the laser-driven source, D. Tupa for the loan of the two titanium-sapphire lasers, C. A. Miller for the loan of the four wire chambers, and our glass blower, W. Lawrence. This work was supported by the U. S. National Science Foundation, the U. S. Department of Energy, the German Bundesministerium für Bildung, Wissenschaft, Forschung und Technologie, and the Polish Committee for Scientific Research. The calculations were performed on the CRAY T90 and T3E at the John von Neumann Institute for Computing, Jülich, Germany.

\section{References}

[1] R. Machleidt, F. Sammarruca, and Y. Song, Phys. Rev. C 53, R1483 (1996).

[2] R. B. Wiringa, V. G. J. Stoks, and R. Schiavilla, Phys. Rev. C 51, 38 (1995).

[3] V. G. J. Stoks, R. A. M. Klomp, C. P. F. Terheggen, and J. J. de Swart, Phys. Rev. C 49, 2950 (1994).

[4] J. L. Friar, G. L. Payne, V. G. J. Stoks, and J. J. de Swart, Phys. Lett. B311, 4 (1993).

[5] A. Nogga, D. Hüber, H. Kamada, and W. Glöckle, Phys. Lett. B409, 19 (1997).

[6] H. Kamada and W. Glöckle, Nucl. Phys. A548, 205 (1992); H. Kamada and W. Glöckle, Few-Body Systems Suppl. 7, 217 (1994); W. Glöckle et al., ibid. 9, 384 (1995); H. Kamada and W. Glöckle, Phys. Rev. Lett. 71, 971 (1993).

[7] S. A. Coon and W. Glöckle, Phys. Rev. C 23, 1790 (1981). 
[8] J. Carlson, V. R. Pandharipande, and R. B. Wiringa, Nucl. Phys. A401, 59 (1983); M. R. Robilotta and M. P. Isidro Filho, ibid. A451, 581 (1986).

[9] R. B. Wiringa, V. Fiks, and A. Fabrocini, Phys. Rev. C 38, 1010 (1988); A. Akmal, V. R. Pandharipande, and D. G. Ravenhall, ibid. 58, 1804 (1998).

[10] J. Fujita and H. Miyazawa, Prog. Theor. Phys. 17, 360 (1957).

[11] J. L. Friar, D. Hüber, and U. van Kolck, Phys. Rev. C 59, 53 (1999).

[12] H. Witała et al., Phys. Rev. Lett. 81, 1183 (1998).

[13] E. J. Stephenson et al., Phys. Rev. C 60, 061001 (1999).

[14] R. E. Adelberger and C. N. Brown, Phys. Rev. D 5, 2139 (1972).

[15] S. P. Wells et al., Nucl. Inst. and Meth. Phys. Res. A 325, 205 (1993).

[16] D. Hüber and J. L. Friar, Phys. Rev. C 58, 674 (1998).

[17] H. Witała, D. Hüber, and W. Glöckle, Phys. Rev. C 49, R14 (1994).

[18] W. Tornow, H. Witała, and A. Kievsky, Phys. Rev. C 57, 555 (1998).

[19] A. Kievsky, Phys. Rev. C 60, 034001 (1999); D. Hüber, J. L. Friar, A. Nogga, H. Witała, and U. van Kolck, nucl-th/9910034.

[20] R. Bieber et al., Phys. Rev. Lett. 84, 606 (2000); H. Sakai et al., ibid. 84, 5288 (2000).

[21] M. Garçon et al., Nucl. Phys. A458, 287 (1986).

[22] H. O. Meyer, Nucl. Phys. A631, 122c (1998).

[23] K. P. Coulter et al., Phys. Rev. Lett. 68, 174 (1992); M. Poelker et al., Nucl. Inst. and Meth. Phys. Res. A 364, 58 (1995); Phys. Rev. A 50, 2450 (1994); M. A. Miller et al., AIP Conf. Proc. 421148 (1998); R. V. Cadman et al., ibid. 421437 (1998).

[24] T. Walker and L. W. Anderson, Nucl. Inst. and Meth. Phys. Res. A 334, 313 (1993).

[25] J. Stenger et al., Phys. Rev. Lett. 78, 4177 (1997).

[26] J. A. Fedchak et al., Nucl. Inst. and Meth. Phys. Res. A 417, 182 (1998).

[27] J. L. Forest (private communication).

[28] D. Hüber, H. Kamada, H. Witała, and W. Glöckle, Acta Phys. Pol. B28, 1677 (1997); W. Glöckle et al., Phys. Rep. 274, 107 (1996).

[29] R. Schiavilla et al., Phys. Rev. C 58, 1263 (1998). 


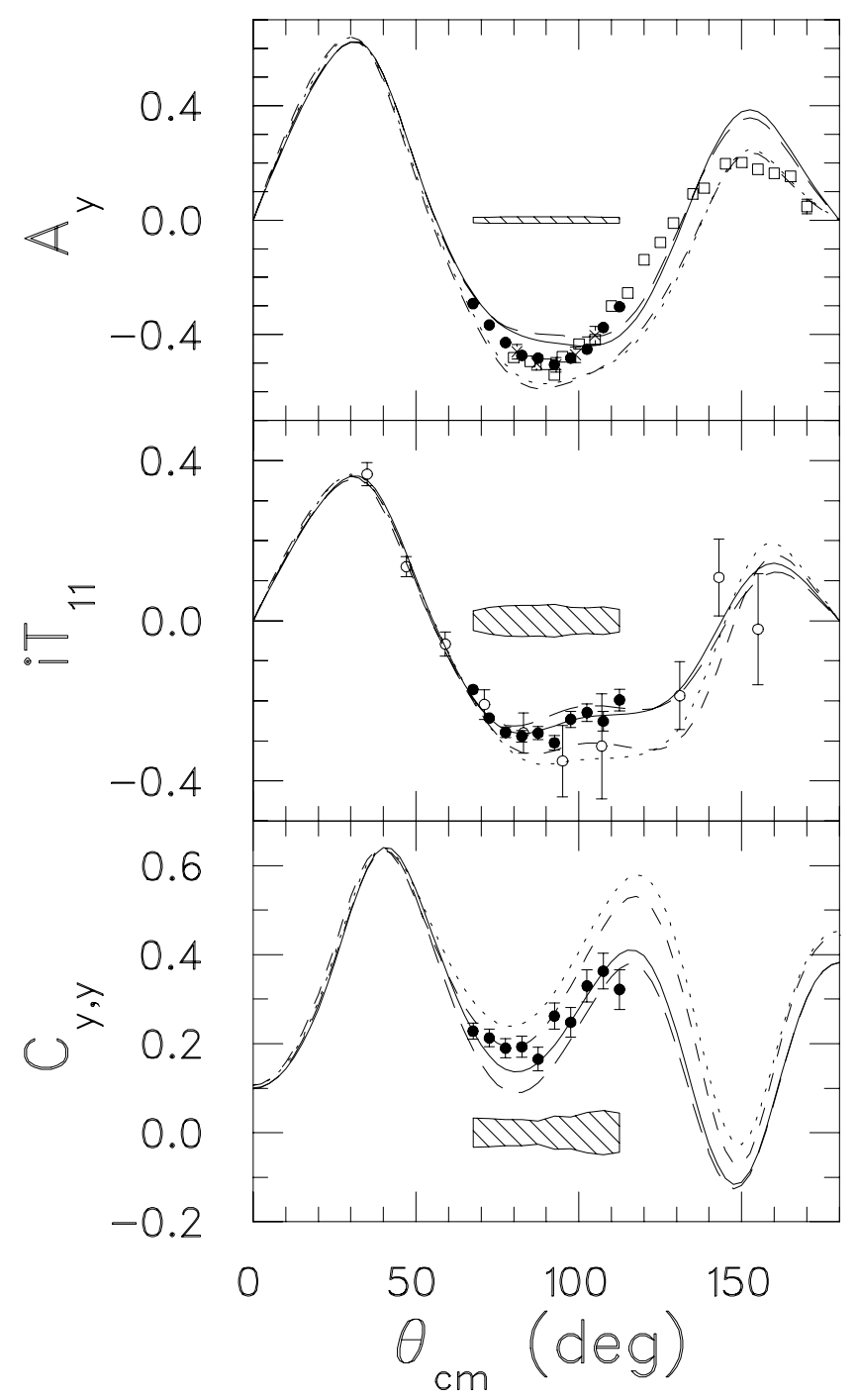

Figure 2: Spin observables in $\vec{p} \vec{d}$ elastic scattering at a proton lab energy of $197 \mathrm{MeV}$ as a function of the proton scattering angle in the center of mass reference frame, $\theta_{\mathrm{cm}}$. The error bars indicate statistical errors and the systematic error is indicated by the hashed area. The top panel shows the the proton analyzing power $A_{y}$, the center panel shows the deuteron vector analyzing power $i T_{11}$, and the bottom panel shows the vector-vector spin correlation parameter $C_{y, y}$. The results of this work are indicated by solid circles. Previous measurements of $A_{y}$ from Rochester (squares) [14] and IUCF (crosses) [15] are also shown, as well as the measurement of $i T_{11}$ with a $395 \mathrm{MeV}$ deuteron beam scattering from a proton target at Saclay (open circles) [21]. The theoretical curves are calculations using CD-Bonn+TM (solid), AV18+TM (long dashed), CD-Bonn only (dotted), and AV18 only (short dashed). 